\section{Risk of Creutzfeldt- Jakob disease transmission by ocular surgery and tissue transplantation}

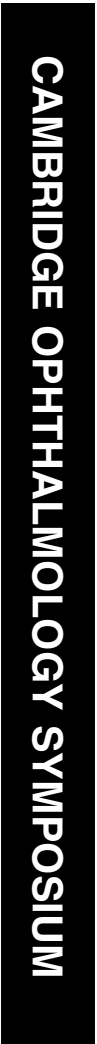

${ }^{1}$ Academic Unit of Ophthalmology, University of Bristol, Bristol, UK

${ }^{2}$ Manchester Royal Eye Hospital, Manchester, UK

${ }^{3}$ National CJD Surveillance Unit, University of Edinburgh, Edinburgh, UK

Correspondence:

WJ Armitage, Academic Unit of Ophthalmology, University of Bristol, Bristol Eye Hospital, Lower Maudlin Street, Bristol BS1 2LX, UK Tel: + 0117342 4585; Fax: + 01179046624 E-mail:w.j.armitage@ bristol.ac.uk

Received: 10 September 2008

Accepted: 30 September 2008

Published online: 9 January 2009
WJ Armitage ${ }^{1}$, AB Tullo² and JW Ironside ${ }^{3}$
Keywords: Creutzfeldt-Jakob disease; prion disease; cornea; retina; transplantation; surgical instruments

Creutzfeldt-Jakob disease (CJD) is a member of the family of prion diseases, all of which are associated with the presence of an abnormal form of the prion protein in the brain. It is a rare, progressive, degenerative disorder of the central nervous system that results in motor dysfunction, dementia, and death. It has an extended asymptomatic incubation period that can last for many years. It occurs in four clinical forms: sporadic (sCJD), genetic (gCJD), iatrogenic (iCJD), and variant (vCJD). ${ }^{1}$

Sporadic CJD, whose cause is unknown, is the most common form, occurring with an annual frequency worldwide of approximately one case per million population and accounting in the United Kingdom for over 75\% of CJD deaths (Figure 1). This form generally affects those aged $60-80$ years and results in a rapidly progressive dementia with death occurring on average within 6 months. The genetic forms of CJD, which are associated with point mutations or insertions in the gene encoding the prion protein (PRNP), are rather rarer (Figure 1). Gerstmann-Sträussler-Scheinker disease (GSS) and fatal familial insomnia (FFI) are also inherited forms of prion disease though phenotypically and genetically distinct from gCJD. Iatrogenic CJD is acquired during medical or surgical treatment and has been responsible for just $4 \%$ of CJD deaths in the United Kingdom since 1995. The other acquired form, vCJD, is considered to be a consequence of human exposure to bovine spongiform encephalopathy (BSE) in the food chain. In contrast to SCJD, vCJD occurs in much younger individuals (median age 26 years; range, 


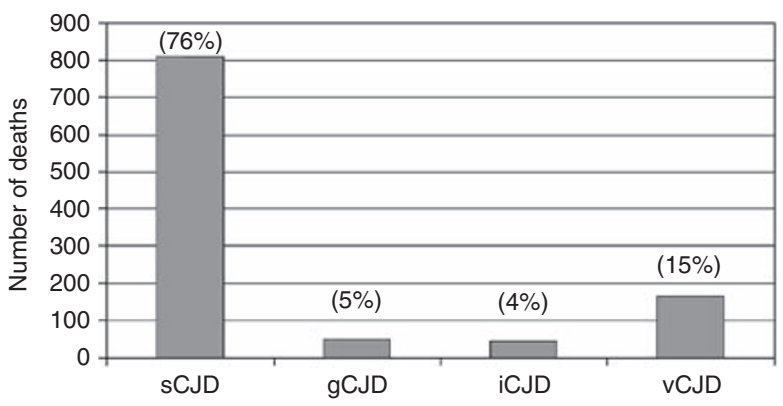

Figure 1 Definite and probable CJD deaths in the United Kingdom from 1995 to September 2008 by CJD type ( $n=1075$; data from the National CJD Surveillance Unit: http://www. cjd.ed.ac.uk).

12-74 years) and the illness has a longer duration (median 14 months; range, 6-40 months). The great majority of cases have occurred in the United Kingdom where there have been 164 vCJD deaths since 1995.

\section{Prion disease}

Transmissible spongiform encephalopathies (TSE) occur in humans (CJD) and other mammalian species, notably scrapie in sheep, BSE in cattle, chronic wasting disease (CWD) in North American cervids, and transmissible mink encephalopathy (TME). These diseases are marked by the transformation of normal prion protein $\left(\mathrm{PrP}^{\mathrm{C}}\right)$, a membrane-bound glycoprotein of unknown physiological function, into a misfolded configuration (PRPS). ${ }^{2}$ This transformation is characterized by a conformational change in protein structure with the refolding of $\alpha$-helices into $\beta$-sheets, which imparts a degree of resistance to proteolytic digestion by proteinase $\mathrm{K}$. Replication of this abnormal form of the protein through progressive conversion of $\operatorname{PrP}^{\mathrm{C}}$, a process that perhaps requires an as yet unidentified cofactor/ molecular chaperon, leads to the accumulation of $\mathrm{PrP}^{\mathrm{Sc}}$ as amyloid fibrils and results in spongiform changes in the brain. According to the prion hypothesis, $\mathrm{PrP}^{\mathrm{Sc}}$ is the sole component of the infectious agent responsible for prion diseases. Although the precise nature of the transmissible agent in prion diseases is uncertain, the presence of $\mathrm{PrP}^{\mathrm{Sc}}$ is currently the only surrogate marker for CJD infectivity in tissues.

\section{$\mathrm{PrP}^{\mathrm{Sc}}$ and sCJD subtypes}

Although $\operatorname{PrP}^{\mathrm{C}}$ is completely degraded by proteolysis, $\mathrm{PrP}^{\mathrm{Sc}}$ is only partially degraded to a proteinase-resistant fragment, $\mathrm{PrP}^{\mathrm{res}}$. Two major subtypes of $\mathrm{PrP}^{\mathrm{res}}$ have been identified by immunoblotting with the relative molecular mass of the unglycosylated band being $21 \mathrm{kD}$ in type 1 and $19 \mathrm{kD}$ in type 2 . The specific methionine $(\mathrm{M}) /$ valine $(\mathrm{V})$

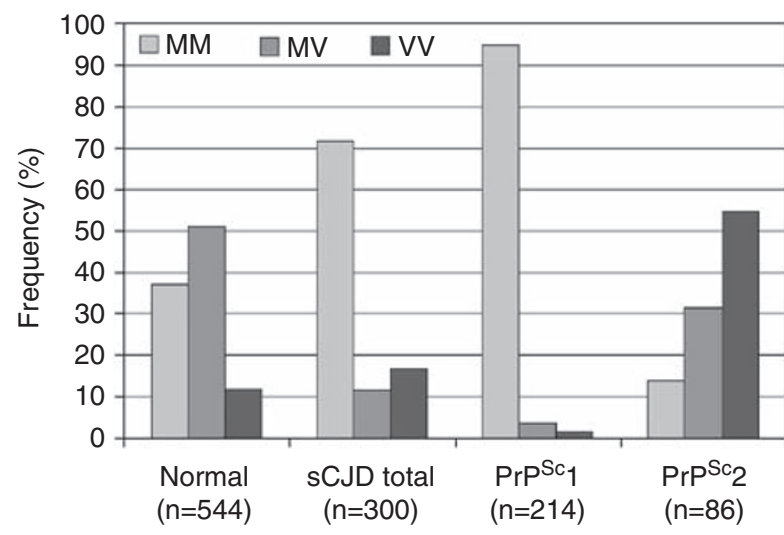

Figure 2 Distribution of codon 129 methionine $(\mathrm{M}) /$ valine $(\mathrm{V})$ polymorphisms of the prion gene (PRNP) in healthy individuals; all cases of sCJD and sCJD broken down by $\mathrm{PrP}^{\mathrm{Sc}}$ subtype (data redrawn from Parchi et $a l^{3}$ with permission of John Wiley \& Sons, Inc.).

polymorphism at codon 129 of $P R N P$, and these two subtypes of $\operatorname{PrP}^{\mathrm{Sc}}$ have been used to define six subtypes of sCJD, viz., MM1, MM2, MV1, MV2, VV1, and VV2. ${ }^{3}$ The frequency distributions of the different codon 129 polymorphisms differ between normal individuals, where $50 \%$ are of the MV phenotype, and those with sCJD where the MM phenotype predominates (Figure 2). Virtually all those with type $1 \mathrm{PrP}^{\mathrm{Sc}}$ are $\mathrm{MM}$, whereas the majority of type $2 \mathrm{PrP}^{\mathrm{Sc}}$ is VV. The clinical course of the disease, including age at onset and duration of disease, onset of dementia, ataxia, myoclonus and visual problems, EEG characteristics and the neuropathological features has been reported to vary with SCJD subtype; for example, the duration of disease in MM1 and MV1 is shorter than for the other subtypes. ${ }^{3}$ A further classification of $\mathrm{PrP}^{\mathrm{Sc}}$ into four biochemically distinct subtypes that are independent of codon 129 polymorphism and PrPres $^{\text {isoform was recently }}$ reported. ${ }^{4}$

\section{Prevalence of vCJD}

Numbers of deaths from vCJD are not necessarily an indicator of the actual prevalence of infected individuals in the population, which includes carriers of the disease that remain asymptomatic and those that are incubating preclinical disease. Susceptibility and the time of onset of clinical disease may also be influenced by gene polymorphisms, which means that the fall in vCJD deaths seen in recent years could be followed by a second peak. A study of 12674 appendectomy and tonsillectomy specimens found three appendix samples with an abnormal accumulation of $\mathrm{PrP}$ - none of the tonsil specimens were positive for $\operatorname{PrP}^{5}$ If it is assumed that all three positives were indicative of $\mathrm{vCJD}$ infection, the 
estimated prevalence in the population of the United Kingdom would be 237 per million (95\% CI, 49-692 per million), which suggests that 3808 individuals (95\% CI, 785-11128) aged 10-30 years are infected but asymptomatic. This report, however, highlights several caveats. ${ }^{5}$ There is uncertainty about the negative results because the rate of accumulation of $\mathrm{PrP}$ in lymphoreticular tissue from the time of infection is not known. Interpretation of the positive results also has a degree of uncertainty and, in an attempt to resolve this, experimental transmission using tissue from one of the positive cases is being investigated. Analysis of the PRNP gene in the remaining two positive cases found that both were valine homozygotes at codon 129 , unlike the clinical cases of vCJD that have thus far all been methionine homozygotes, suggesting that this genotype is also susceptible to BSE infection. ${ }^{6}$ Because of continuing concerns about the potential human-human transmission of vCJD, a large scale study utilizing the National Anonymous Tonsil Archive (NATA) to examine 100000 pairs of tonsils collected during routine tonsillectomies is currently underway.

\section{Iatrogenic CJD}

Worldwide data quoted by the World Health Organization (WHO) in 2006 show that there have been at least 363 iCJD deaths. ${ }^{1,7}$ Apart from seven deaths where neurosurgical instruments or stereotactic EEG electrodes were implicated in the transmission and two instances of transmission by corneal allograft (one confirmed, one probable - there are also a number of possible transmissions), most iCJD has resulted from the use of human pituitary-derived hormones (growth hormone, 180 deaths; gonadotropin, four deaths) and dura mater allografts (168 deaths). There have also been four reports of transmission of vCJD by blood transfusion $^{8}$ (these are classified as vCJD rather than iCJD deaths in the National CJD Surveillance Unit data shown in Figure 1).

\section{Risk of CJD transmission by ocular tissue}

\section{Retina and optic nerve}

The latest WHO classification of TSE infectivity ${ }^{1}$ puts retina and optic nerve into the high infectivity category as infection has been transmitted to non-human primates by ocular tissue homogenates from humans that died of CJD. ${ }^{9}$ There are two reports of detection of $\mathrm{PrP}^{\mathrm{Sc}}$ in retina and optic nerve of eyes from individuals with vCJD, although there are quantitative differences in the findings. Wadsworth et $a l^{10}$ found the levels of $\mathrm{PrP}^{\mathrm{Sc}}$ relative to brain from the same individual of $2.5 \%$ in retina and $25 \%$ in optic nerve, whereas Head et $a l^{11}$ found similar levels of $\mathrm{PrP}^{\mathrm{Sc}}$ in retina and brain with lower levels in optic nerve. The findings of these two groups also diverged over the detection of ocular $\mathrm{PrP}^{\mathrm{Sc}}$ in SCJD individuals, with one group reporting similarly high levels of $\operatorname{PrP}^{\mathrm{Sc}}$ in retina and brain ${ }^{11}$ in contrast to the failure of the other group to detect $\mathrm{PrP}^{\mathrm{Sc}}$ in retina. ${ }^{10} \mathrm{An}$ explanation for these discrepancies based on differences in disease progression between sCJD subtypes and vCJD has been proposed by Head et al. ${ }^{12}$ It is likely that $\operatorname{PrP}^{\mathrm{Sc}}$ accumulates in the retina by centrifugal spread from the brain along the optic nerve, which suggests that the amount and distribution of $\mathrm{PrP}^{\mathrm{Sc}}$ in the retina could be a function of the duration of the disease. Both groups were able to detect $\mathrm{PrP}^{\mathrm{Sc}}$ in retinas from vCJD individuals presumably owing to the longer duration of disease compared with SCJD. Moreover, the sCJD individual in whose retina $\operatorname{PrP}^{\mathrm{Sc}}$ was not detected had the MM1 subtype, which has a shorter duration than the less common VV2 subtype of the individual where retinal $\mathrm{PrP}^{\mathrm{Sc}}$ was not detected. Head et al ${ }^{12}$ subsequently had the opportunity also to study retina from an individual with subtype MM1 sCJD. They did this time detect retinal $\operatorname{PrP}^{\mathrm{Sc}}$, but only after phospotungstate precipitation and at a level that was between 0.1 and $1 \%$ of that in vCJD brain. Overall, these biochemical studies and the transmission studies in non-human primates indicate that retina and optic nerve do have the potential to transmit CJD infection and are accordingly included in the high infectivity category of the WHO classification. ${ }^{1}$

\section{Cornea and anterior segment}

Cornea is classified by WHO in the lower infectivity category because $\mathrm{PrP}^{\mathrm{Sc}}$ has not been detected by biochemical or immunohistochemical means in any ocular tissue other than retina and optic nerve. ${ }^{10,11}$ Penetrating keratoplasty (PK) in non-human primates and guinea pigs using corneas from sCJD-infected individuals has not transmitted CJD; but placing infected tissue into the anterior chamber of guinea pigs or injecting emulsified corneal tissue into the brains of mice did result in the appearance of clinical/pathological signs of CJD in some but not all of the recipients. ${ }^{13}$

There have been several reports of corneal allograft recipients subsequently developing SCJD. ${ }^{14,15}$ The first of these was in 1974 and is the only one considered to be a confirmed case of iatrogenic transmission as there was autopsy confirmation of CJD in both the donor and recipient. ${ }^{16}$ This was also the first reported humanhuman transmission of sCJD. Only one of the other cases is thought to be a probable transmission, ${ }^{17}$ whereas the remaining reports are speculative owing to the lack of autopsy confirmation of CJD in the eye donors. ${ }^{14}$ Indeed, 
modelling and population studies strongly suggest that these cases could be coincidental infections rather than iatrogenic transmissions of CJD. More than 30000 corneal transplants are carried out every year in the United States, and it has been estimated that one case of sCJD could occur coincidentally in this population every 18 months. ${ }^{15}$

By contrast, an incident occurred in 1997 in the United Kingdom where sCJD was confirmed in an eye donor after the corneas and sclerae had been transplanted, but before the recipients had developed any related symptoms. Shortly before the 56-year-old female donor died from carcinoma of the bronchus, she had developed neurological symptoms thought to be due to brain metastases. However, on postmortem examination, this turned out not to be the case, and a diagnosis of sCJD was later confirmed. ${ }^{14}$ The recipients were advised that the tissue should be removed, which was accepted by one of the cornea recipients and by the single recipient of both sclerae, but not by the recipient of the fellow cornea. Immunohistochemical staining of the removed tissue failed to detect $\mathrm{PrP}^{\mathrm{Sc}}$. The cornea recipient who opted to have the tissue removed died at the age of 92 years due to bronchopneumonia 7 years after the initial PK, showing signs of dementia not considered consistent with iCJD. The other two recipients were still free of any symptoms suggestive of iCJD 8 years after the event. ${ }^{14}$

\section{Reducing risk of transmission by tissue allografts}

A validated test for CJD infectivity in tissue donors is not currently available for routine use. Apart from questions about the most suitable analyte (e.g. tonsil, spleen, or retina), the interpretation of positive and negative results is still uncertain. The outcome of a test could be influenced by considerations such as the CJD subtype and whether sufficient $\mathrm{PrP}^{\mathrm{Sc}}$ accumulates in tissue during incubation of the disease to allow detection.

Application of appropriate donor selection criteria to exclude ocular tissue donors that could possibly be harbouring CJD remains an important means of assuring the safety of corneal and limbal tissue/cell transplants. These criteria were thoroughly reviewed in the United Kingdom following the 1997 CJD incident mentioned earlier. ${ }^{14}$ In fact, if the extant criteria at the time had been applied correctly, the eye donation would not have taken place (i.e. the donor had a neurological condition of unknown aetiology, presumed to be metastases to the brain, that had to be investigated by postmortem examination). There must, however, be a balance to achieve an acceptable degree of risk reduction without excluding large numbers of potential donors because selection criteria are too restrictive. ${ }^{18}$ The effectiveness of donor selection criteria is supported by the data from the
United States where hundreds of thousands of corneal transplants have taken place since the case of iCJD in $1974^{16}$ without any further reports of confirmed or probable transmissions of CJD.

\section{Risk of CJD transmission by surgical instruments}

The infectious agent of CJD adheres strongly to metal, and experiments with metal wires exposed briefly to infected tissue have shown this to be a highly effective route of transmission of infection. ${ }^{19}$ Concerns have therefore been raised about transmission by surgical instruments given that the infectious agent is remarkably resistant to conventional means of instrument sterilization. These concerns were heightened by the finding of $\mathrm{PrP}^{\mathrm{Sc}}$ in peripheral tissues not just from individuals with vCJD but from those with SCJD and iCJD. ${ }^{20}$

In the United Kingdom, single-use instruments are used for eye retrieval and all tissue processing by the main eye banks. This renders cross-contamination between donors, not just of CJD, unlikely. Although epidemiological evidence fails to support transmission of CJD by ophthalmic surgical instruments, the possibility of iCJD by this route cannot be excluded. ${ }^{21,22}$ In the absence of single-use alternatives for many ophthalmic instruments, the tracking of instruments and the avoidance of migration between instrument sets are important safeguards to prevent the onward transmission of CJD, in the event that specific instruments need to be identified and quarantined. It is also important to follow recommended protocols for the cleaning and disinfection of instruments. The very small number of reported transmissions of CJD by neurosurgical instruments is noteworthy in this respect. ${ }^{7}$ Advice on surgical instruments and on the risk of CJD transmission is available from the Royal College of Ophthalmologists (http://www.rcophth.ac.uk).

\section{Conclusions}

Ocular tissue clearly has the potential to transmit CJD, though the risk is likely to be substantially different for the various component tissues of the eye with retina and optic nerve posing the greatest risk. There is one confirmed and one probable case of iatrogenic CJD transmission by corneal transplantation, as well as a few reported possible transmissions that could have been coincidental infections. A validated test for CJD in human tissue donors is not currently available; however, selection criteria that exclude donors that may be infected by CJD would appear to have been effective. The risk of transmission posed by surgical instruments is likely to be low given the very few transmissions reported following neurosurgery; but this route of 
transmission cannot be excluded. In the absence of single-use alternatives, the application of recommended cleaning and disinfection protocols and the ability to identify and quarantine instruments that may have been used on an infected patient are important means of risk reduction.

\section{Acknowledgements}

This paper was presented at the Cambridge Ophthalmological Symposium 2008. Proprietary interests: None

\section{References}

1 WHO guidelines on tissue infectivity distribution in transmissible spongiform encephalopathies, World Health Organization: Geneva, 2006.

2 Prusiner SB. Prions. Proc Nat Acad Sci 1998; 95: 13363-13383.

3 Parchi P, Giese A, Capellari S, Brown P, Schulz-Schaeffer W, Windl $\mathrm{O}$ et al. Classification of sporadic Creutzfeldt-Jakob disease based on molecular and phenotypic analysis of 300 subjects. Ann Neurol 1999; 46: 224-233.

4 Uro-Coste E, Cassard H, Simon S, Lugan S, Bilheude J-M, Perret-Liaudet A et al. Beyond PrPres) type 1/type 2 dichotomy in Creutzfeldt-Jakob disease. PLoS Pathogens 2008; 4: e1000029.

5 Hilton DA, Ghani AC, Conyers L, Edwards P, McCardle L, Ritchie D et al. Prevalence of lymphoreticular prion protein accumulation in UK tissue samples. J Pathol 2004; 203: 733-739.

6 Ironside JW, Bishop MT, Connolly K, Hegazy D, Lowrie S, Le Grice $\mathrm{M}$ et al. Variant Creutzfeldt-Jakob disease: prion protein genotype analysis of positive appendix tissue samples from a retrospective prevalence study. BMJ 2006; 332: 1186-1188.

7 Brown P, Preece M, Brandel JP, Sato T, McShane L, Zerr I et al. Iatrogenic Creutzfeldt-Jakob disease at the millennium. Neurology 2000; 55: 1075-1081.

8 Brown P. Creutzfeldt-Jakob disease: reflections on the risk from blood product therapy. Haemophilia 2007; 13(Suppl 5): 33-40.

9 Brown P, Gibbs Jr CJ, Rodgers-Johnson P, Asher DM, Sulima MP, Bacote A et al. Human spongiform encephalopathy: the National Institutes of Health series of 300 cases of experimentally transmitted disease. Ann Neurol 1994; 35: 513-529.

10 Wadsworth JD, Joiner S, Hill AF, Campbell TA, Desbruslais $\mathrm{M}$, Luthert PJ et al. Tissue distribution of protease resistant prion protein in variant Creutzfeldt-Jakob disease using a highly sensitive immunoblotting assay. Lancet 2001; 358: 171-180.

11 Head MW, Northcott V, Rennison K, Ritchie D, McCardle L, Bunn TJR et al. Prion protein accumulation in eyes of patients with sporadic and variant Creutzfeldt-Jakob disease. Invest Ophthalmol Vis Sci 2003; 44: 342-346.

12 Head MW, Peden AH, Yull HM, Ritchie DL, Bonshek RE, Tullo $\mathrm{AB}$ et al. Abnormal prion protein in the retina of the most commonly occurring subtype of sporadic CreutzfeldtJakob disease. Br J Ophthalmol 2005; 89: 1131-1133.

13 Hogan RN, Cavanagh HD. Transplantation of corneal tissue from donors with diseases of the central nervous system. Cornea 1995; 14: 547-553.

14 Tullo AB, Buckley RJ, Kelly T, Head MW, Bennett P, Armitage WJ et al. Transplantation of ocular tissue from a donor with sporadic Creutzfeldt-Jakob disease. Clin Exp Ophthalmol 2006; 34: 645-649.

15 Maddox RA, Belay ED, Curns AT, Zou W-Q, Nowicki S, Lembach RG et al. Creutzfeldt-Jakob disease in recipients of corneal transplants. Cornea 2008; 27: 851-854.

16 Duffy P, Wolf J, Collins G, DeVoe AG, Streeten B, Cowen D. Possible person-to-person transmission of CreutzfeldtJakob disease. N Engl J Med 1974; 290: 692-693.

17 Heckmann JG, Lang CJ, Petruch F, Druschky A, Erb C, Brown P et al. Transmission of Creutzfeldt-Jakob disease via a corneal transplant. J Neurol Neurosurg Psychiatry 1997; 63: 388-390.

18 Kennedy RH, Hogan RN, Brown P, Holland E, Johnson RT, Stark W et al. Eye banking and screening for CreutzfeldtJakob disease. Arch Ophthalmol 2001; 119: 721-726.

19 Weissmann C, Enari M, Klohn PC, Rossi D, Flechsig E. Transmission of prions. J Infect Dis 2002; 186(Suppl 2): S157-S165.

20 Peden AH, Ritchie DL, Head MW, Ironside JW. Detection and localization of $\mathrm{PrP}^{\mathrm{Sc}}$ in the skeletal muscle of patients with variant, iatrogenic, and sporadic forms of CreutzfeldtJakob disease. Am J Pathol 2006; 168: 927-935.

21 Ward HJT, Everington D, Cousens SN, Smith-Bathgate B, Gillies M, Murray K et al. Risk factors for sporadic Creutzfeldt-Jakob disease. Ann Neurol 2008; 63: 347-354.

22 S-Juan P, Ward HJT, De Silva R, Knight RSG, Will RG. Ophthalmic surgery and Creutzfeldt-Jakob disease. Br J Ophthalmol 2004; 88: 446-449. 